\title{
Astaxanthin ameliorates renal interstitial fibrosis and peritubular capillary rarefaction in unilateral ureteral obstruction
}

\author{
JIN ZHAO, MEIXIA MENG, JINHUA ZHANG, LILI LI, \\ XIAOJING ZHU, LI ZHANG, CHANG WANG and MING GAO \\ Department of Nephrology, Xi'an No. 4 Hospital, Xi'an, Shaanxi 710004, P.R. China
}

Received July 18, 2018; Accepted January 29, 2019

DOI: $10.3892 / \mathrm{mmr} .2019 .9970$

\begin{abstract}
Loss of peritubular capillaries is a notable feature of progressive renal interstitial fibrosis. Astaxanthin (ASX) is a natural carotenoid with various biological activities. The present study aimed to evaluate the effect of ASX on unilateral ureteral obstruction (UUO)-induced renal fibrosis in mice. For that purpose, mice were randomly divided into five treatment groups: Sham, ASX 100 mg/kg, UUO, UUO + ASX 50 mg/kg and UUO + ASX $100 \mathrm{mg} / \mathrm{kg}$. ASX was administered to the mice for 7 or 14 days following UUO. The results demonstrated that UUO-induced histopathological changes in the kidney tissue were prevented by ASX. Renal function was improved by ASX treatment, as evidenced by decreased blood urea nitrogen and serum creatinine levels. Furthermore, the extent of renal fibrosis and collagen deposition induced by UUO was suppressed by ASX. The levels of collagen I, fibronectin and $\alpha$-smooth muscle actin were increased by UUO in mice or by transforming growth factor (TGF)- $\beta 1$ treatment in NRK-52E cells, and were reduced by ASX administration. In addition, ASX inhibited the UUO-induced decrease in peritubular capillary density by upregulating vascular endothelial growth factor and downregulating thrombospondin 1 levels. Inactivation of the TGF- $\beta 1 / \mathrm{Smad}$ signaling pathway was involved in the anti-fibrotic mechanism of ASX in UUO mice and TGF- $\beta 1$-treated NRK-52E cells. In conclusion, ASX attenuated renal interstitial fibrosis and peritubular capillary rarefaction via inactivation of the TGF- $\beta 1 / \mathrm{Smad}$ signaling pathway.
\end{abstract}

\section{Introduction}

Renal interstitial fibrosis is one of the main pathological features of chronic kidney disease (1). As the disease

Correspondence to: Dr Jin Zhao, Department of Nephrology, Xi'an No. 4 Hospital, 21 Jiefang Road, Xi'an, Shaanxi 710004, P.R. China E-mail: zhaojin201808@163.com

Key words: astaxanthin, renal interstitial fibrosis, peritubular capillaries, VEGF, TSP-1 progresses, patients may suffer glomerular or tubulointerstitial lesions, which may result in end-stage renal failure and even mortality (2). At present, there are limited treatments available for alleviating renal fibrosis. Therefore, the identification of more effective methods for preventing or decelerating the progression of renal fibrosis is urgently required.

Loss of peritubular capillaries has been regarded as the distinguishing feature of progressive interstitial fibrosis (3-5). Increasing evidence has demonstrated that renal fibrosis is associated with peritubular capillary loss in experimental animals and clinical patients $(6,7)$. It has been demonstrated that renal injury may block peritubular capillaries and then cause hypoxic injury, which stimulates interstitial fibrosis (8-10). In addition, the loss of capillaries has also been confirmed to induce fibrosis under certain conditions, such as glomerulonephritis and unilateral ureteral obstruction (UUO) $(11,12)$. Thus, replacing damaged capillary vessels is an important approach to mitigating interstitial fibrosis, and angiogenesis serves a crucial role in this process (12-14). Vascular endothelial growth factor (VEGF) is important in promoting the formation of tubule-like structures by endothelial cells and is an essential mediator of angiogenesis (15). A previous study demonstrated that thrombospondin 1 (TSP-1), an anti-angiogenic molecule, could further worsen progressive renal disease (16). Downregulated VEGF expression and increased TSP-1 expression are closely associated with renal interstitial fibrosis (4). In addition, TSP-1 has been confirmed to induce the activation of transforming growth factor (TGF)- $\beta 1$, which is a key cytokine in the induction of renal interstitial fibrosis (17).

Astaxanthin (ASX) is a natural carotenoid and a major source of red pigments in marine animals. Growing evidence suggests that ASX has various biological activities, including antioxidant, anti-inflammatory, ultraviolet ray-resistance, anti-tumor and immune regulatory effects (18-21). A previous study revealed that ASX could prevent capillary regression in atrophied soleus muscles by upregulating VEGF and downregulating TSP-1 (22). However, whether ASX has a protective effect against renal interstitial fibrosis via regulation of VEGF and TSP-1 remains unclear.

In the present study, a renal interstitial fibrosis model was established by UUO in mice. The effects of ASX on UUO-induced renal interstitial fibrosis and its potential mechanisms were investigated. 


\section{Materials and methods}

Animals and experimental protocol. Adult male C57BL/6J mice ( $\mathrm{n}=30$; 6-8 weeks) weighing 20-22 $\mathrm{g}$ were purchased from Beijing HFK Bioscience Co., Ltd. (Beijing, China) and maintained at $21-23^{\circ} \mathrm{C}$ with $45-55 \%$ humidity in a $12 \mathrm{~h}$ light/dark cycle, with ad libitum access to food and water. Mice were randomly divided into five groups ( $n=6 /$ group): Sham, ASX $100 \mathrm{mg} / \mathrm{kg}$, UUO, UUO + ASX $50 \mathrm{mg} / \mathrm{kg}$ and UUO + ASX $100 \mathrm{mg} / \mathrm{kg}$. The doses of ASX were selected according to previous studies $(23,24)$. Renal interstitial fibrosis was induced by UUO, as previously described (25). Briefly, the mice were anesthetized by intraperitoneal injection of pentobarbital sodium $(50 \mathrm{mg} / \mathrm{kg})$. The right ureter was exposed and ligated. The mice in the sham group were subjected to the same operation, but without ureter ligation. Following surgery, the mice in the ASX groups were treated with 50 or $100 \mathrm{mg} / \mathrm{kg}$ ASX (cat. no. A141428; Shanghai Aladdin Biochemical Technology Co., Ltd., Shanghai, China) once daily by oral gavage for 7 or 14 days. The mice in the other groups were treated with the same volume of normal saline. Blood samples were collected from the mouse eye socket 7 or 14 days after the operation, and the mice were then sacrificed by cervical dislocation. Kidney tissues were frozen in liquid nitrogen and stored at $-80^{\circ} \mathrm{C}$ or fixed in $4 \%$ paraformaldehyde at room temperature until use. The animals were treated in accordance with the National Institutes of Health Guide for the Care and Use of Laboratory Animals (26) guidelines. All animal protocols used in the present study were approved by the Institutional Animal Care and Use Committee of Xi'an No. 4 Hospital (Xi'an, China).

Biochemical determinations. The levels of blood urea nitrogen (BUN; cat. no. C013-2) and serum creatinine $(\mathrm{Cr})$ were detected with commercial kits (BUN; cat. no. C011-2; Nanjing Jiancheng Bioengineering Institute, Nanjing, China), according to the manufacturer's instructions.

Histological examination. Kidney tissues fixed in $4 \%$ paraformaldehyde were washed with water, dehydrated by a graded ethanol series (70, 80, 90 and 100\%) and embedded in paraffin. Then, the paraffin-embedded specimens were cut into $5 \mu \mathrm{m}$-thick sections. To observe the pathological changes in renal tissues, the sections were subjected to periodic acid-Schiff (PAS) staining for $15 \mathrm{~min}$ at room temperature and scored on a scale from 0 to 4 ( 0 , no changes; 1 , changes affecting $<25 \%$ of the section; 2, changes affecting $25-50 \%$ of the section; 3 , changes affecting $50-75 \%$ of the section; and 4 , changes affecting $75-100 \%$ of the section) (27). Collagen deposition in renal tissues was evaluated by Masson's trichrome staining and graded as follows: 0 , no staining; $1,<25 \%$ staining of the section; 2, 25-50\% staining of the section; 3, 50-75\% staining of the section; and 4, 75-100\% staining of the section (27). The tissue sections were visualized and photographed under a light microscope (Olympus Corporation, Tokyo, Japan) at x200 magnification.

Immunohistochemical staining. The $5 \mu \mathrm{m}$-thick paraffinembedded renal tissue sections were subjected to immunohistochemical staining. Following deparaffinization with xylene and rehydration in a graded ethanol series $(95,85$ and $75 \%$ ), the sections were heated at $100^{\circ} \mathrm{C}$ in the presence of sodium citrate antigen retrieval solution in a microwave oven for $10 \mathrm{~min}$. Then, the sections were incubated with $10 \% \mathrm{H}_{2} \mathrm{O}_{2}$ for $15 \mathrm{~min}$ at room temperature and blocked with $10 \%$ goat serum (Beijing Solarbio Science \& Technology Co., Ltd., Beijing, China) for $15 \mathrm{~min}$ at room temperature. Subsequently, the sections were incubated with primary antibodies against collagen I (1:100; cat. no. BA0325; Boster Biological Technology, Pleasanton, CA, USA), TSP-1 (1:50; cat. no. 18304-1-AP; ProteinTech Group, Inc., Chicago, IL, USA), VEGF-A (1:50; cat. no. 19003-1-AP; ProteinTech Group, Inc.) and cluster of differentiation 34 (CD34) (1:50; cat. no. 14486-1-AP; ProteinTech Group, Inc.) overnight at $4{ }^{\circ} \mathrm{C}$, followed by incubation with biotin-labeled goat anti-rabbit immunoglobulin G (IgG) (1:200; cat. no. A0277; Beyotime Institute of Biotechnology, Haimen, China) at $37^{\circ} \mathrm{C}$ for $30 \mathrm{~min}$. Then, the sections were incubated with horseradish peroxidase (HRP)-labeled streptavidin (Beyotime Institute of Biotechnology), stained with a DAB Substrate kit (cat. no. DA1010; Beijing Solarbio Science \& Technology Co., Ltd.) and counterstained with hematoxylin for $3 \mathrm{~min}$ at room temperature. The stained sections were observed under a light microscope and photographed at magnification $\mathrm{x} 400$. The results were analyzed by ImageJ 1.8.0 software (National Institutes of Health, Bethesda, MD, USA).

Cell culture and treatment. Rat NRK-52E cells were purchased from Procell (Wuhan, China) and cultured in Dulbecco's modified Eagle's medium supplemented with $5 \%$ fetal bovine serum (Biological Industries, Kibbutz Beit-Haemek, Israel) at $37^{\circ} \mathrm{C}$ in $5 \% \mathrm{CO}_{2}$. To investigate the beneficial effect of ASX in vitro, NRK-52E cells at $70 \%$ confluence were treated with ASX $(10 \mu \mathrm{M})$ in combination with recombinant TGF- $\beta 1$ (5 ng/ml, Wuhan USCN Business Co., Ltd., Wuhan, China) for $72 \mathrm{~h}$ at $37^{\circ} \mathrm{C}$. The dose of ASX was selected according to a previous study (28).

Western blotting. Protein was extracted from renal tissues and NRK-52E cells using radioimmunoprecipitation assay lysis buffer (Beyotime Institute of Biotechnology) containing $1 \%$ phenylmethylsulfonyl fluoride (Beyotime Institute of Biotechnology). The Enhanced BCA Protein Assay kit (Beyotime Institute of Biotechnology) was used to determine protein concentration. A total of $40 \mu \mathrm{g}$ protein was subjected to SDS-PAGE (8 or 10\% gel) and transferred onto polyvinylidene fluoride membranes. After blocking with 5\% skimmed milk for $1 \mathrm{~h}$ at room temperature, the membranes were probed with primary antibodies against collagen I (1:2,000; cat. no. 14695-1-AP; ProteinTech Group, Inc.), TSP-1 (1:500; cat. no. 18304-1-AP; ProteinTech Group, Inc.), VEGF-A (1:1,000; cat. no. 19003-1-AP; ProteinTech Group, Inc.), TGF- $\beta 1$ (1:500; cat. no. 21898-1-AP; ProteinTech Group, Inc.), phosphorylated (p)-Smad2 (1:1,000; cat. no. 3108; Cell Signaling Technology, Inc., Danvers, MA, USA), Smad2 (1:3,000; cat. no. 12570-1-AP; ProteinTech Group, Inc.), fibronectin (1:1,000; cat. no. 15613-1-AP; ProteinTech Group, Inc.), $\alpha$-smooth muscle actin ( $\alpha$-SMA) (1:500; cat. no. 55135-1-AP; ProteinTech Group, Inc.), Smad3 (1:1,000; cat. no. bs-3484R; BIOSS, Beijing, China), p-Smad3 (1:1,000; cat. no. bsm-52205R; BIOSS), Smad4 (1:1,000; cat. no. bs-0585R; 

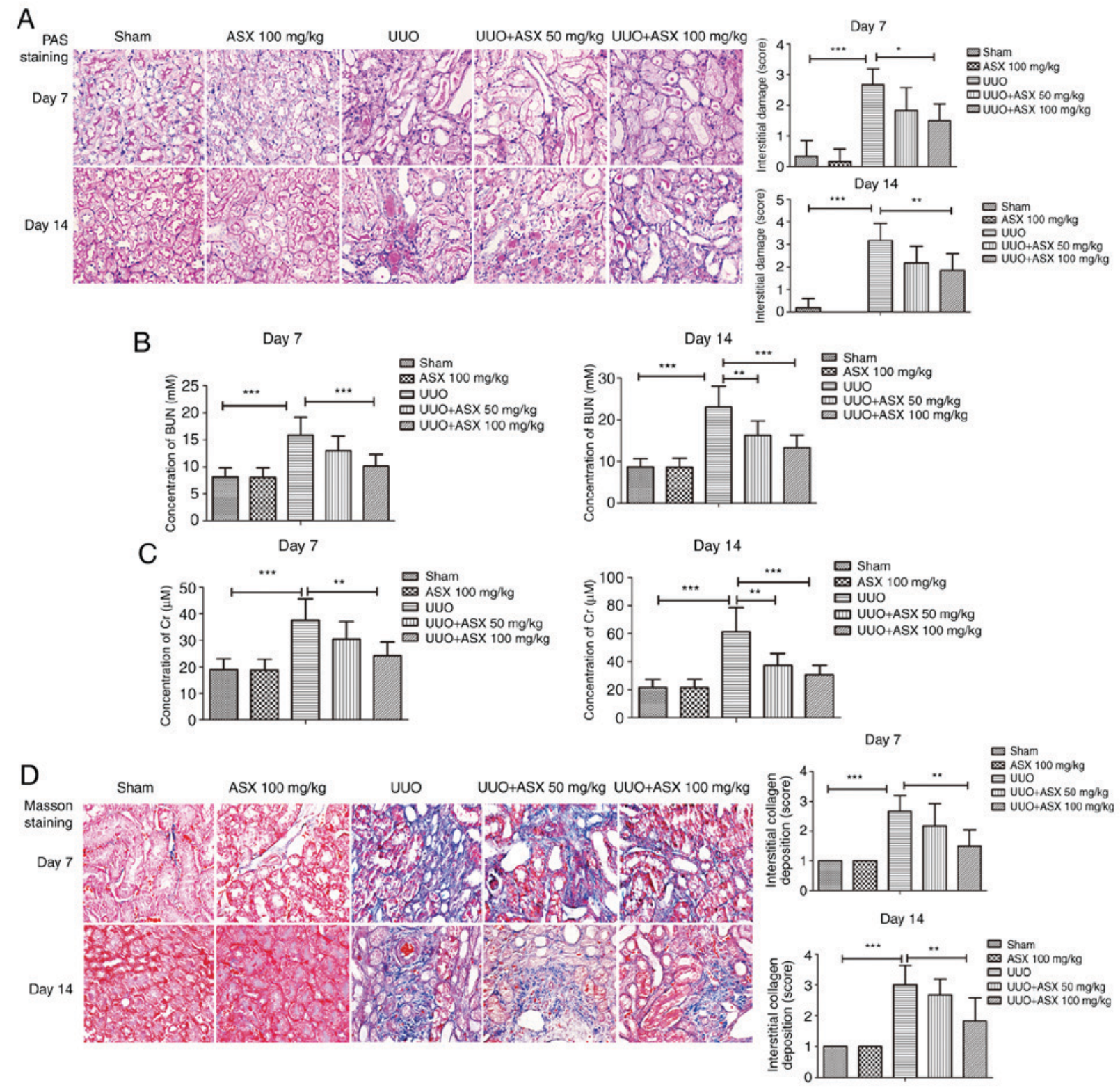

Figure 1. ASX alleviates UUO-induced renal injury in mice. (A) PAS staining was performed to evaluate the histopathological changes in murine renal tissues (magnification, $\mathrm{x} 400$ ). (B) BUN and (C) serum Cr concentrations were measured to assess renal function. (D) Collagen deposition and renal fibrosis were detected by Masson's trichrome staining (magnification, $\mathrm{x} 400)$. The data are presented as the means \pm standard deviation $(\mathrm{n}=6)$. ${ }^{*} \mathrm{P}<0.05,{ }^{* *} \mathrm{P}<0.01,{ }^{* * * *} \mathrm{P}<0.001$. ASX, Astaxanthin; BUN, blood urea nitrogen; Cr, creatinine; PAS, periodic acid-Schiff; UUO, unilateral ureteral obstruction.

BIOSS), Smad7 (1:1,000; cat. no. bs-23328R; BIOSS) and $\beta$-actin (1:500; cat. no. bsm-33036M; BIOSS) overnight at $4^{\circ} \mathrm{C}$. Then, the membrane was incubated with HRP-labeled goat anti-rabbit IgG (cat. no. A0208) or goat anti-mouse IgG (cat. no. A0216; both 1:5,000; Beyotime Institute of Biotechnology) at $37^{\circ} \mathrm{C}$ for $45 \mathrm{~min}$. Proteins were visualized using BeyoECL Plus (Beyotime Institute of Biotechnology). Densitometric analysis was performed using Gel-Pro Analyzer 4 software (Media Cybernetics, Inc., Rockville, MD, USA).

RNA isolation and reverse transcription-quantitative polymerase chain reaction ( $R T-q P C R)$. Total RNA was isolated from renal tissues using a Total RNA Isolation kit (cat. no. RP1001; BioTeke Corporation, Beijing, China). RT was carried out using Super M-MLV Reverse Transcriptase with buffer (cat. no. PR6502; BioTeke Corporation), oligo (dT)15 primers (cat. no. C1101-20; Promega Corporation, Madison, WI, USA), dNTP (cat. no. PR3001, BioTeke Corporation) at $25^{\circ} \mathrm{C}$ for $10 \mathrm{~min}, 42^{\circ} \mathrm{C}$ for $50 \mathrm{~min}$, and then $80^{\circ} \mathrm{C}$ for $10 \mathrm{~min}$. qPCR was performed on an Exicycler ${ }^{\mathrm{TM}} 96$ Real-Time Quantitative Thermal Block (Bioneer Corporation, Daejeon, Korea) using the 2X Power Taq PCR Master mix (cat. no. PR1702; BioTeke Corporation) and SYBR Green (cat. no. SY1020; Beijing Solarbio Science \& Technology Co., Ltd.). The following primers were used: Collagen I (forward, 5'-GGA CGCCATCAAGGTCTACT-3' and reverse, 5'-GAATCCATC GGTCATGCTCT-3'); TSP-1 (forward, 5'-GACCAGAGGGAC ACGGACAT-3' and reverse, 5'-TGGCATTAGGCACATAGG GA-3'); VEGF-A (forward, 5'-CGTGAGCCCTCCCCCTTG-3' and reverse, 5'-GCCCAGAAGTTGGACGAAAA-3'); and $\beta$-actin (forward, 5'-CTGTGCCCATCTACGAGGGCTAT-3' and reverse, 5'-TTTGATGTCACGCACGATTTCC-3'). The 

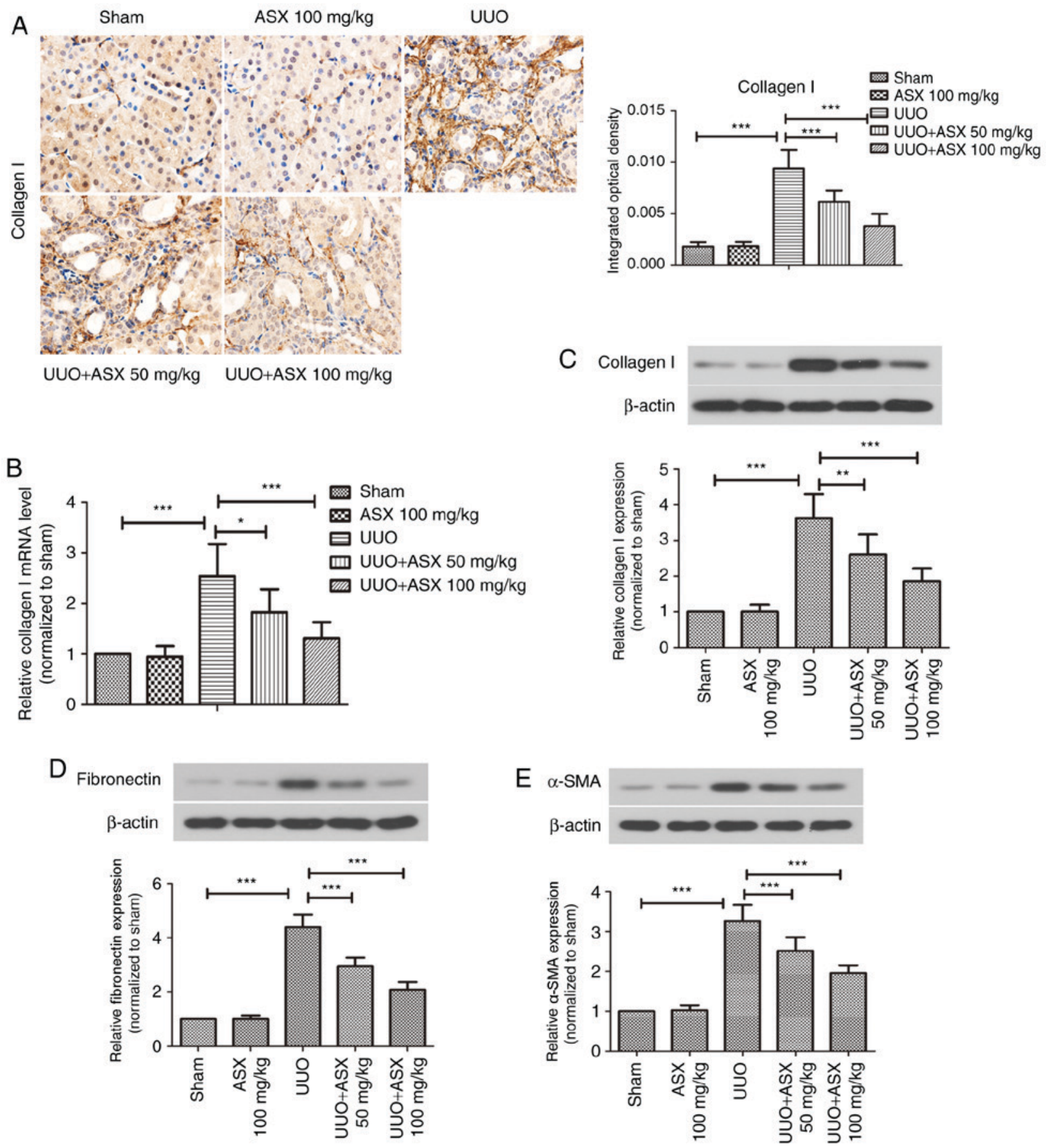

Figure 2. ASX suppresses collagen expression and renal fibrosis in mice. (A) Expression of collagen I in murine renal tissues was assessed by immunohistochemical staining (magnification, x600). (B) mRNA levels of collagen I were determined by reverse transcription-quantitative polymerase chain reaction. Western blot analysis was used to assess the protein expression levels of (C) collagen I, (D) fibronectin, and (E) $\alpha$-SMA. The data are presented as the means \pm standard deviation $(\mathrm{n}=6)$. ${ }^{*} \mathrm{P}<0.05,{ }^{* *} \mathrm{P}<0.01,{ }^{* * *} \mathrm{P}<0.001$. $\alpha$-SMA, $\alpha$-smooth muscle actin; ASX, Astaxanthin; UUO, unilateral ureteral obstruction.

thermocycling conditions were as follows: Denaturation for 5 min at $95^{\circ} \mathrm{C}$, followed by 40 cycles of $10 \mathrm{sec}$ at $94^{\circ} \mathrm{C}, 20 \mathrm{sec}$ at $60^{\circ} \mathrm{C}$ and $30 \mathrm{sec}$ at $72^{\circ} \mathrm{C}$. The relative mRNA levels were calculated using the $2^{-\Delta \Delta \mathrm{Cq}}$ method (29).

Statistical analysis. All experimental data are presented as the means \pm standard deviation of three experimental repeats. Comparisons among different experimental groups were performed using one-way analysis of variance followed by Bonferroni multiple comparisons test using GraphPad Prism 5 software (GraphPad Software, Inc., La Jolla, CA,
USA). $\mathrm{P}<0.05$ was considered to indicate a statistically significant difference.

\section{Results}

ASX alleviates UUO-induced renal injury in mice. Histopathological changes in the kidneys were determined by PAS staining. As shown in Fig. 1A, UUO induced significant interstitial damage in mouse kidneys on day 7 and 14, which could be relieved by treatment with ASX. Furthermore, the concentration of BUN and serum $\mathrm{Cr}$ were significantly 
A

A Sham ASX $100 \mathrm{mg} / \mathrm{kg} \quad$ UUO
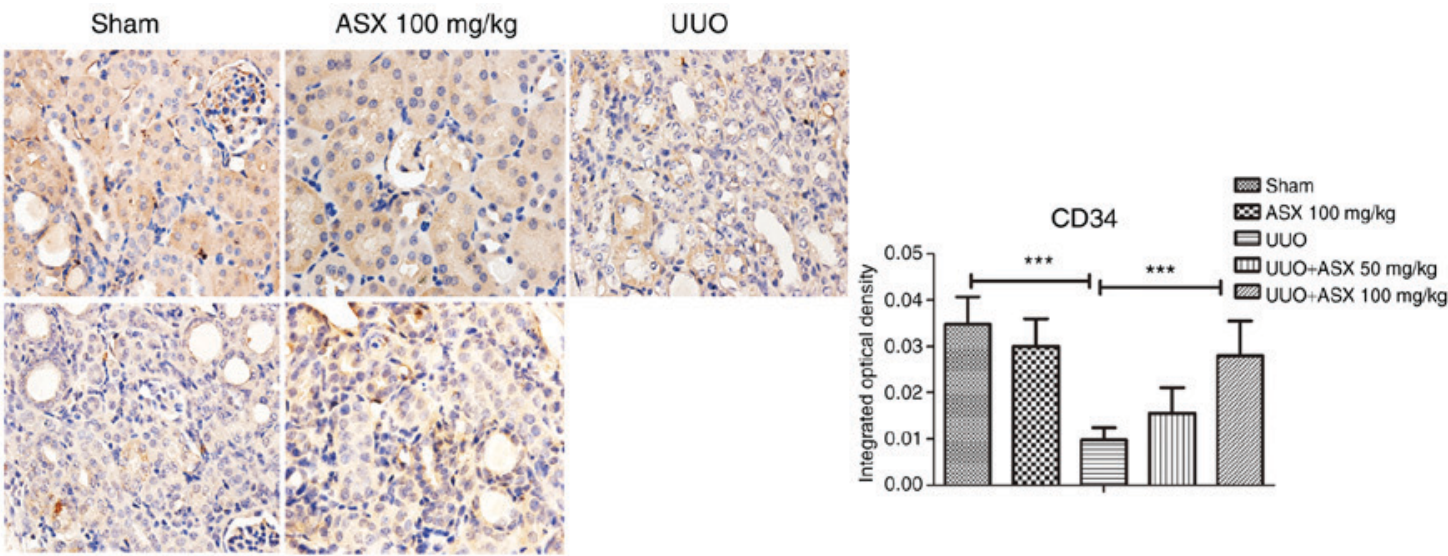

UUO+ASX $50 \mathrm{mg} / \mathrm{kg} \quad$ UUO+ASX $100 \mathrm{mg} / \mathrm{kg}$
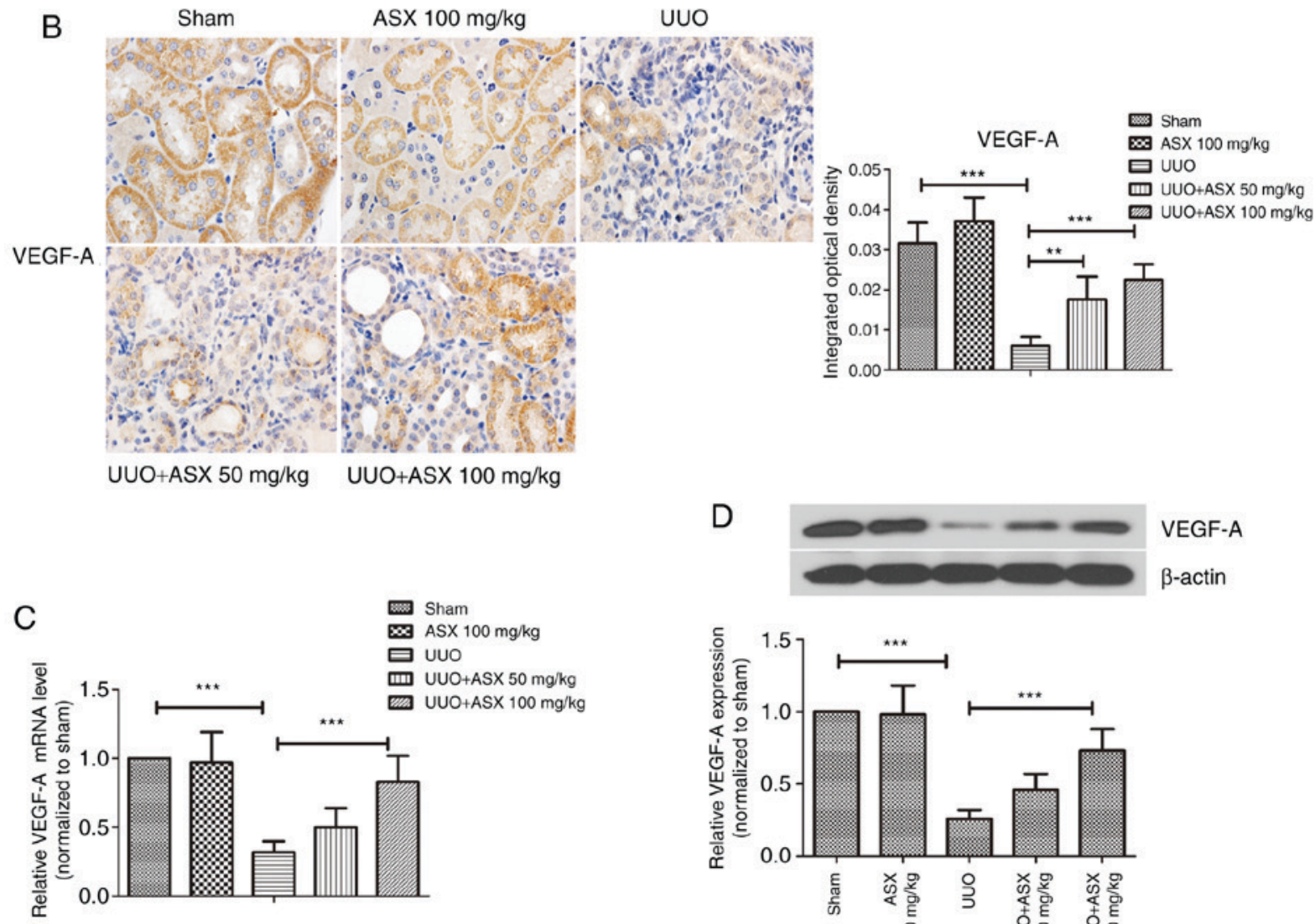

$\mathrm{D}$

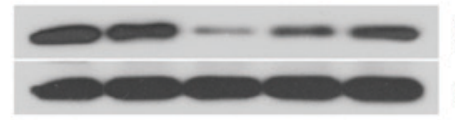

VEGF-A

$\beta$-actin

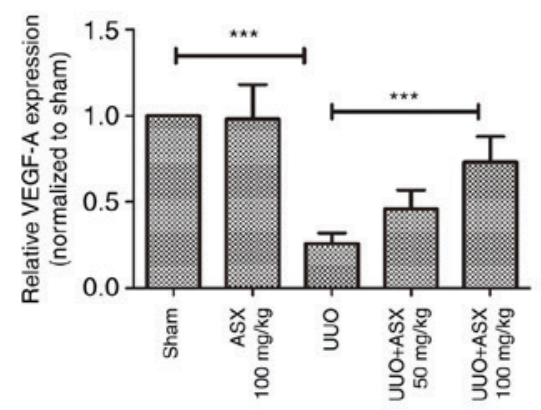

Figure 3. ASX increases the density of peritubular capillaries and VEGF-A expression. (A) Density of peritubular capillaries was evaluated by immunohistochemical staining of CD34 (magnification, x600). (B) Expression of VEGF-A in murine renal tissues was determined by immunohistochemical staining (magnification, x600). (C) mRNA levels of VEGF-A were detected by reverse transcription-quantitative polymerase chain reaction. (D) Protein expression levels of VEGF-A were assessed by western blotting. The data are presented as the means \pm standard deviation $(\mathrm{n}=6) .{ }^{* *} \mathrm{P}<0.01,{ }^{* * * *} \mathrm{P}<0.001$. ASX, Astaxanthin; CD34, cluster of differentiation 34; UUO, unilateral ureteral obstruction; VEGF-A, vascular endothelial growth factor A.

increased in the UUO group on day 7 and 14 (Fig. 1B and C), which was consistent with previous studies and indicated a deterioration of renal function $(30,31)$. However, ASX treatment markedly reduced the UUO-induced BUN and serum $\mathrm{Cr}$ levels (Fig. 1B and C). These results indicated that treatment with ASX alleviated UUO-induced renal injury and improved renal function.

ASX suppresses collagen formation and renal fibrosis in mice. To observe collagen deposition and renal fibrosis, Masson's trichrome staining was performed. As presented in Fig. 1D,
UUO treatment resulted in significantly increased interstitial collagen deposition on day 7 and 14 and obvious renal fibrosis, which could be suppressed by ASX administration. In addition, the expression of collagen I in renal tissues was evaluated by immunohistochemical staining. As shown in Fig. 2A, a significant increase in collagen I staining in renal tissues was observed in the UUO group on day 14, whereas ASX treatment effectively inhibited UUO-induced collagen I expression. RT-qPCR and western blot analysis further demonstrated that ASX prevented the UUO-mediated increase in mRNA and protein levels of collagen I in renal tissues on day 14 , in 

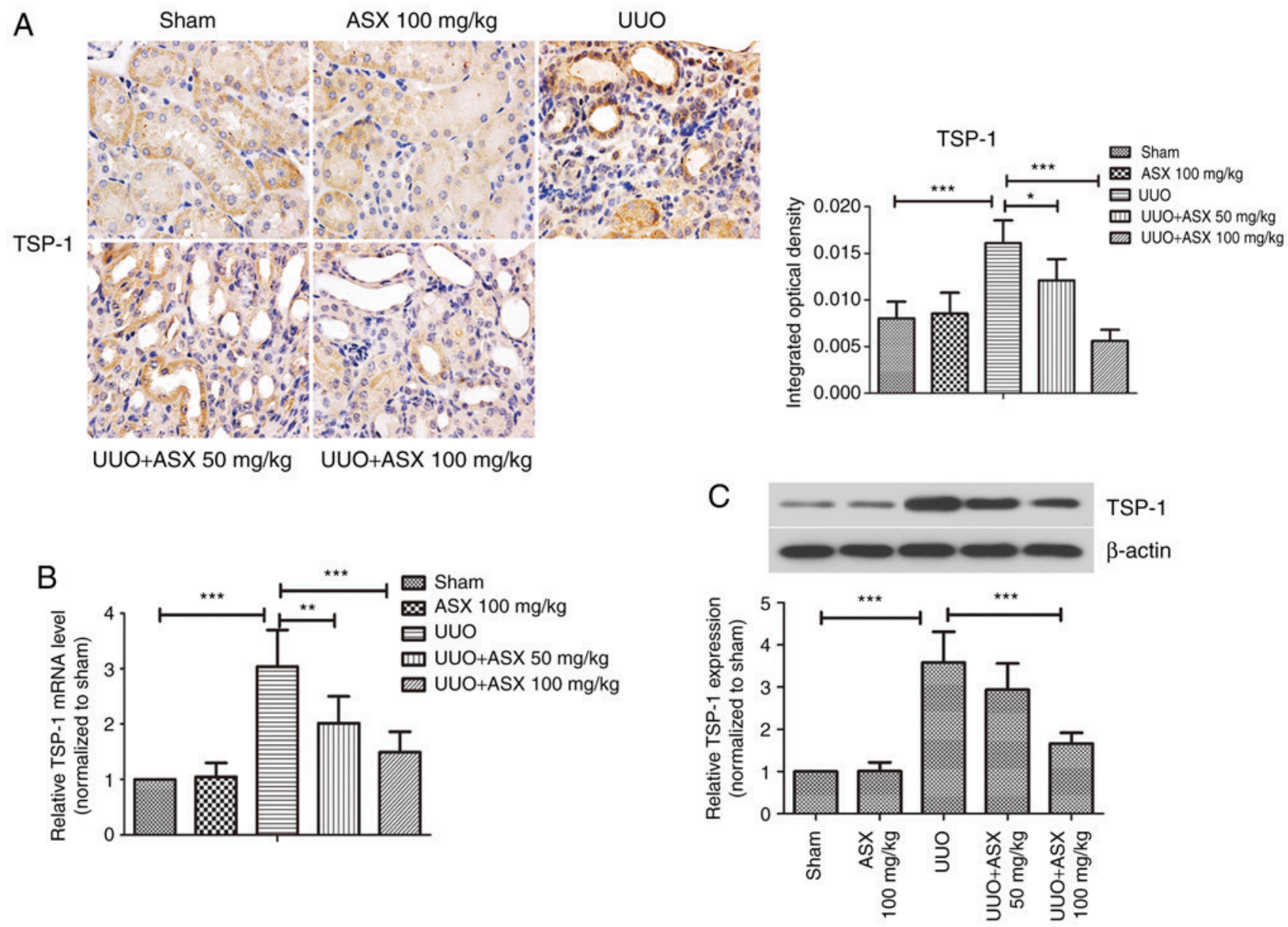

Figure 4. ASX inhibits TSP-1 expression in renal fibrotic tissue. (A) Expression of TSP-1 in murine renal tissues was assessed by immunohistochemical staining (magnification, x600). (B) mRNA levels of TSP-1 were detected by reverse transcription-quantitative polymerase chain reaction. (C) Protein expression levels of TSP-1 were assessed by western blotting. The data are presented as the means \pm standard deviation $(\mathrm{n}=6)$. ${ }^{*} \mathrm{P}<0.05,{ }^{* * *} \mathrm{P}<0.01,{ }^{* * * *} \mathrm{P}<0.001$. ASX, Astaxanthin; TSP-1, thrombospondin 1; UUO, unilateral ureteral obstruction.

a dose-dependent manner (Fig. 2B and C). In addition, the protein levels of fibronectin and $\alpha$-SMA were increased in the renal tissues of UUO-treated mice, and were suppressed by ASX administration (Fig. 2D and E). These findings suggested that ASX mitigated UUO-induced renal interstitial fibrosis in mice.

ASX enhances the density of peritubular capillaries and VEGF-A expression. Peritubular capillaries were observed by immunohistochemical staining of CD34, a typical marker of endothelial cells. As presented in Fig. 3A, peritubular capillaries could be easily recognized in the sham and ASX control groups due to CD34 immunostaining. However, UUO treatment led to a decrease in CD34-positive capillaries in renal tissues on day 14, which was mitigated when ASX was administered. The expression of VEGF-A, an important mediator of angiogenesis, was next studied. As shown in Fig. 3B, the positive immunohistochemical staining of VEGF-A was markedly reduced by UUO on day 14 . Renal tissues exhibited significantly stronger staining of VEGF-A in the ASX treatment groups compared with the UUO group. In addition, the mRNA and protein levels of VEGF-A in renal tissues were decreased by $\sim 70 \%$ in the UUO group, and this was reversed by ASX in a dose-dependent manner (Fig. 3C and D). These results suggested that ASX prevented the UUO-induced decrease in density of peritubular capillaries by upregulating VEGF-A.

ASX inhibits TSP-1 expression in renal fibrotic tissues. The expression of the anti-angiogenic factor TSP-1 was detected by immunohistochemical staining, as shown in Fig. 4A. Positive staining for TSP-1 was most pronounced in the UUO group on day 14, which was significantly reduced by ASX administration. Consistently, the mRNA and protein expression levels of TSP-1 in renal tissues were increased by $\sim 3$-fold in the UUO group on day 14, while they were markedly repressed by treatment with ASX (Fig. 4B and C). Thus, regulation of TSP-1 expression may be a potential mechanism underlying the beneficial effects of ASX and targeting this protein could be useful for treating renal fibrosis.

ASX prevents UUO-induced activation of the TGF- $\beta 1 /$ Smad signaling pathway. The TGF- $\beta 1 /$ Smad signaling pathway serves pivotal roles in renal fibrosis (32). Thus, the present study investigated the effect of ASX on activation of TGF- $\beta 1$ and Smad2/3/4/7. As illustrated in Fig. 5A-D, the protein expression levels of TGF- $\beta 1, \mathrm{p}-\mathrm{Smad} 2, \mathrm{p}$-Smad 3 as well as Smad4, were significantly increased by UUO on day 14 , while they could be significantly suppressed by ASX treatment. The 
A
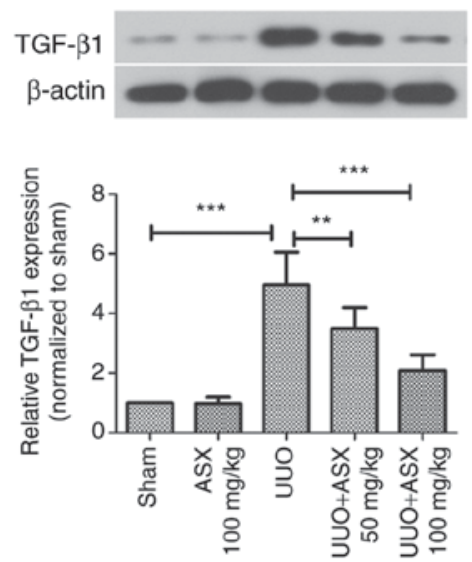

B
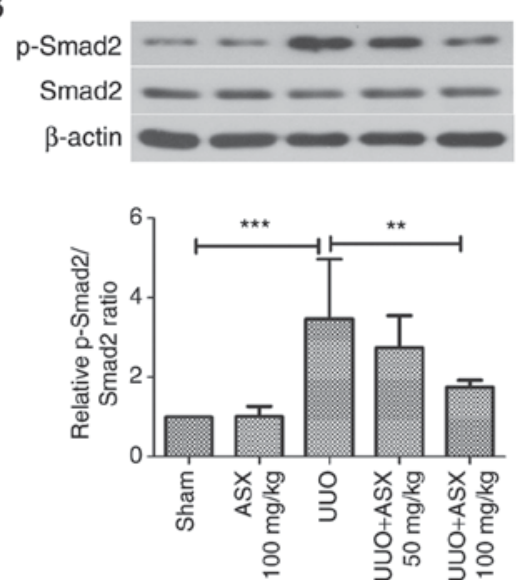

C
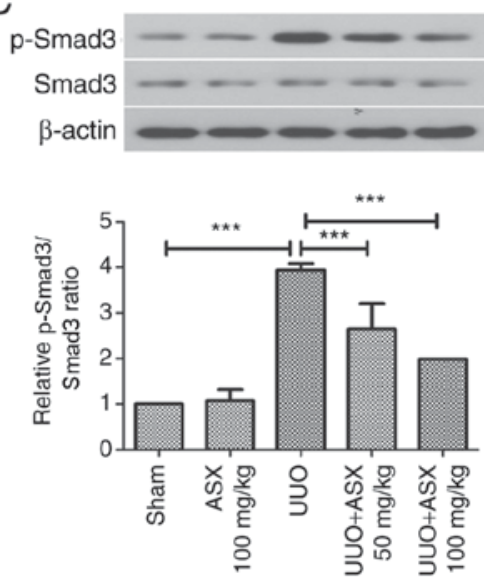

$D_{\text {Smad4 }}$ $\beta$-actin
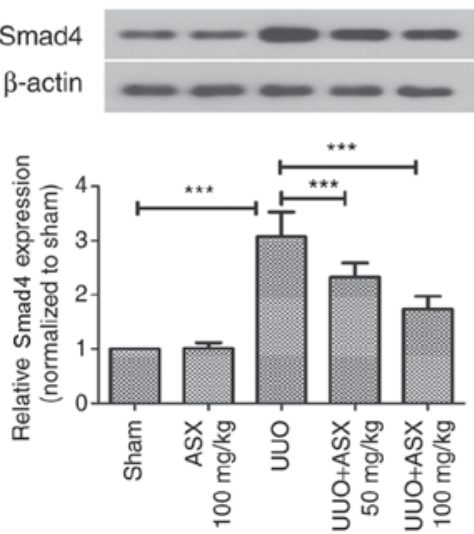

E
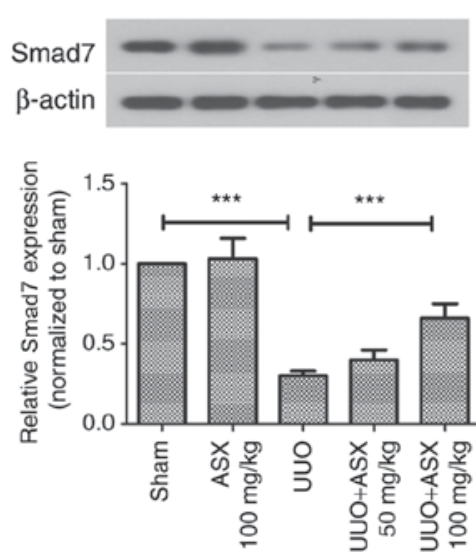

Figure 5. ASX inhibits UUO-induced activation of the TGF- $\beta 1 /$ Smad signaling pathway. Protein expression levels of (A) TGF- $\beta 1$, (B) Smad2, (C) Smad3, (D) Smad4 and (E) Smad7 in murine renal tissues were assessed by western blotting. The data are presented as the means \pm standard deviation $(\mathrm{n}=6)$. ${ }^{* *} \mathrm{P}<0.01$, ${ }^{* * *} \mathrm{P}<0.001$. ASX, Astaxanthin; p, phosphorylated; TGF- $\beta 1$, transforming growth factor $\beta 1$; UUO, unilateral ureteral obstruction.

protein expression level of Smad7, which is inhibitory, was decreased by UUO, which was enhanced by ASX administration (Fig. 5E). Thus, inactivation of the TGF- $\beta 1 / \mathrm{Smad}$ signaling pathway appeared to be involved in the protective mechanism of ASX.

ASX suppresses TGF- $\beta 1$-induced expression of profibrogenic factors via inactivation of the Smad signaling pathway. The present study next investigated the in vitro effects of ASX on the expression of pro-fibrotic factors in NRK-52E cells. As shown in Fig. 6A and B, the protein expression levels of collagen I and $\alpha$-SMA were significantly increased upon stimulation with TGF- $\beta 1$ in NRK-52E cells. However, this increase in $\alpha-S M A$ and collagen I levels was suppressed by ASX. Furthermore, the present study examined whether ASX treatment affected the Smad signaling pathway in vitro. As illustrated in Fig. 6 C and D, treatment with ASX attenuated the phosphorylation of Smad2 and Smad3 in TGF- $\beta 1$-stimulated NRK-52E cells. However, TGF- $\beta 1$ and ASX treatment had no effect on the expression of Smad4 or Smad7 proteins in NRK-52E cells (Fig. 6E and F).

\section{Discussion}

The present study investigated the role of ASX in UUO-induced renal fibrosis in mice. The results revealed that ASX treatment effectively ameliorated UUO-induced renal injury and dysfunction, inhibited renal fibrosis and collagen deposition, and enhanced the density of peritubular capillaries by upregulating VEGF-A and downregulating TSP-1 expression levels. Inactivation of the TGF- $\beta 1 /$ Smad signaling pathway appeared to be involved in the protective effect of ASX.

Renal injury was determined on day 7 and day 14 following UUO in the present study. Previous studies which evaluated renal injury on different days post-UUO, indicated that kidney fibrosis tends to get worse with time $(33,34)$. Consistently, the present results revealed that UUO-induced renal damage was exacerbated with the prolonging of time, as evidenced by PAS staining and increased BUN and serum $\mathrm{Cr}$ levels. However, there was very little difference in the collagen deposition assessed by Masson's trichrome staining between day 7 and day 14. ASX treatment for both 7 and 14 days could alleviate UUO-induced renal damage, but longer treatment seemed to be more effective.

Renal fibrosis is a pathological process that contributes to chronic renal failure $(2,35)$. Accumulation of excessive extracellular matrix is an important feature of renal interstitial fibrosis (36). Mammals have a high collagen content, with over 27 types of collagen that make up $\sim 30 \%$ of the overall protein (37). Collagen type I (also known as collagen I) is regarded as a matrix component and one of the fibril-forming collagens (38). Previous studies revealed that inhibition of collagen I expression could attenuate fibrosis formation 
A

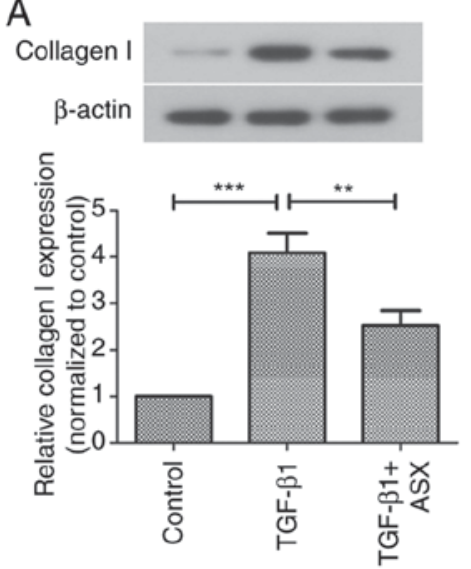

D
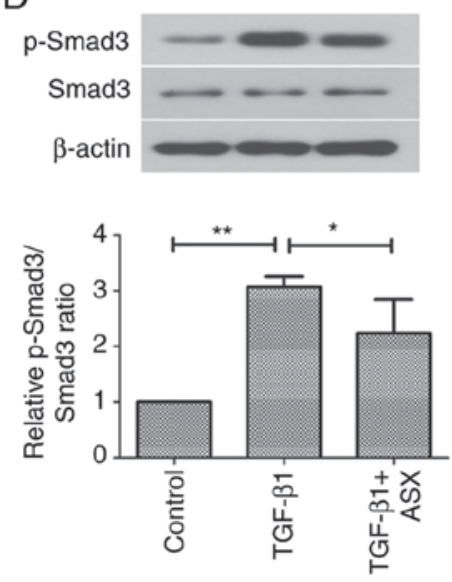

B
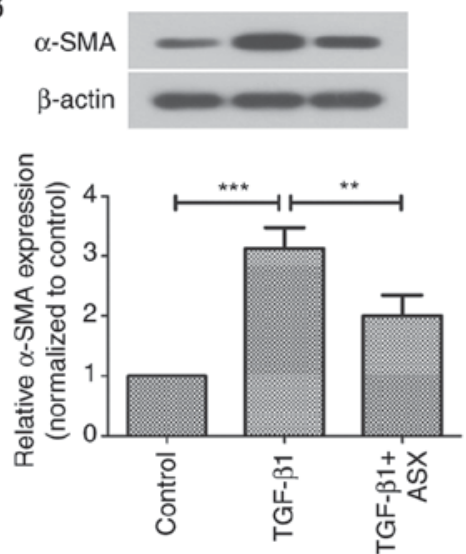

E
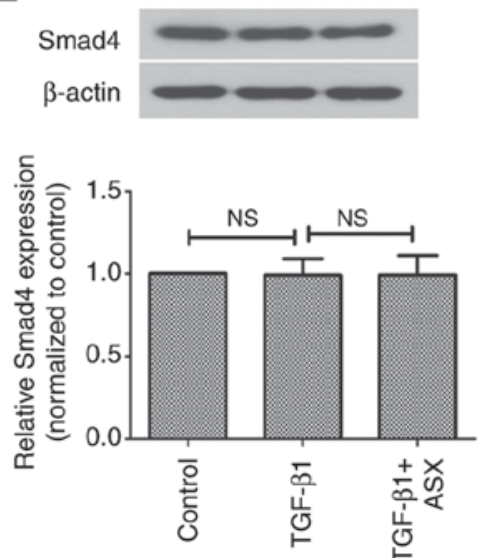

C
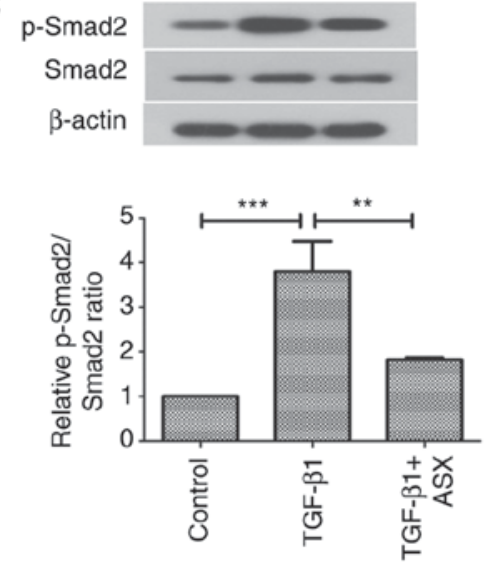

F
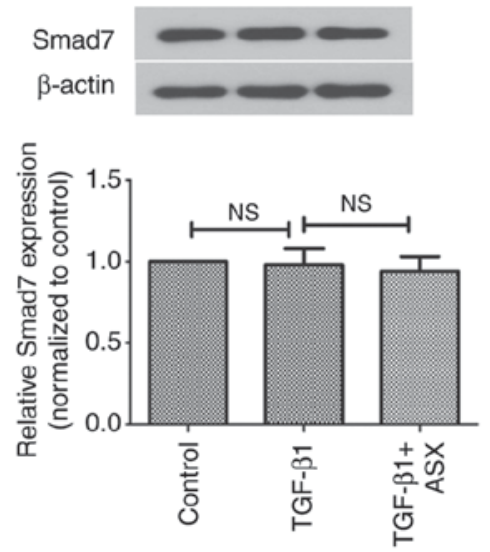

Figure 6. ASX suppresses TGF- $\beta 1$-induced expression of profibrogenic factors via inactivation of the Smad signaling pathway. Protein expression levels of (A) collagen I, (B) $\alpha$-SMA, (C) Smad2, (D) Smad3, (E) Smad4 and (F) Smad7 in NRK-52E cells were assessed by western blotting. The data are presented as the means \pm standard deviation $(\mathrm{n}=6) .{ }^{*} \mathrm{P}<0.05,{ }^{* *} \mathrm{P}<0.01,{ }^{* * *} \mathrm{P}<0.001$. ASX, Astaxanthin; NS, not significant; $\mathrm{p}$, phosphorylated; TGF- $\beta 1$, transforming growth factor $\beta 1$; UUO, unilateral ureteral obstruction.

in various organs, including the liver and kidney $(39,40)$. Fibronectin and collagen I are extracellular matrix components, and serve crucial roles in fibrosis of the kidney and eventual nephropathy $(41,42)$. $\alpha$-SMA is a marker of activated myofibroblasts, which is upregulated by UUO (43). According to the present results, ASX significantly alleviated UUO-induced renal fibrosis and collagen deposition, as confirmed by downregulation of the protein expression levels of collagen I, fibronectin and $\alpha$-SMA.

It has been well documented that peritubular capillaries transport oxygen and nutrients to renal tubular and interstitial cells, serving a pivotal role in sustaining normal hemodynamics and kidney function (44). Therefore, chronic tubular hypoxia may occur due to decreased density of peritubular capillaries, which further promotes extracellular matrix synthesis and contributes to the progression of tubulointerstitial fibrosis (44). The loss of peritubular capillaries has been verified in patients with interstitial fibrosis and animal models (5). In the present study, reduced density of peritubular capillaries was observed in UUO-treated mice, which was significantly increased by ASX treatment. Furthermore, the potential mechanisms of ASX in regulating the density of peritubular capillaries were explored. Since angiogenesis serves a key role in this process, the present study investigated whether the expression of angiogenic or anti-angiogenic factors was altered. VEGF is an important angiogenic factor. A previous study reported that VEGF was markedly downregulated in the tubules of rats with interstitial fibrosis (45). The loss of VEGF has also been verified in human patients with renal interstitial fibrosis (46). Thus, reduced VEGF expression appears to be involved in the pathological mechanism of renal interstitial fibrosis. The present study also observed that UUO treatment led to decreased VEGF-A expression, which was reversed by ASX treatment.

Next, the present study focused on TSP-1, which is an anti-angiogenic factor and an endogenous activator of TGF- $\beta 1(47,48)$. It has been previously demonstrated that TSP-1 is associated with the loss of microvascular endothelium (49). In addition, TSP-1 can exert anti-angiogenic effects by preventing VEGF-induced proliferation of endothelial cells and inducing endothelial cell apoptosis $(50,51)$. Sun et al (52) suggested that suppression of TSP-1 expression enhances the density of peritubular capillaries and ameliorates tubulointerstitial fibrosis. Apart from its role in reducing peritubular capillary density, TSP-1-mediated activation of TGF- $\beta 1$ serves crucial roles in promoting fibrosis in multiple organs (53-55). Thus, TSP-1 may be used as a therapeutic target for renal fibrosis. In the present study, as expected, administration of ASX inhibited the UUO-induced increase in TSP-1 expression. Therefore, 
it is likely that ASX attenuated the UUO-induced loss of peritubular capillaries by upregulating VEGF-A and downregulating TSP-1.

To investigate the underlying mechanisms of ASX in renal fibrosis, the TGF- $\beta /$ Smad signaling pathway was explored. The activation of the TGF- $\beta 1$ signaling pathway has been demonstrated to serve key roles in the progression of renal fibrosis $(32,56)$. TGF- $\beta 1$ facilitates the protein synthesis of collagen I and promotes extracellular matrix accumulation via the Smad signaling pathway $(57,58)$. The most important Smad proteins for TGF- $\beta 1$ signal transduction are Smad2, Smad3 and Smad4 as well as the inhibitor, Smad7. The activation of Smad 2 and Smad 3 is induced by TGF- $\beta 1$. Subsequently, the Smad complex is formed by the binding of $\mathrm{p}-\mathrm{Smad} 2, \mathrm{p}$-Smad3 and Smad4, which in turn migrates to the nucleus and regulates the transcription of target genes (59). Smad7, an inhibitory Smad protein, suppresses the activation and phosphorylation of $S m a d 2 / 3$. A previous study demonstrated the role of Smad2 in the pro-fibrotic TGF- $\beta 1$ signaling pathway (60). Smad2/3 was confirmed to be activated in the context of renal interstitial fibrosis in various chronic kidney diseases (61). In addition, silencing of Smad3 suppressed the progression of renal fibrosis in mice $(62,63)$. In the present study, ASX effectively suppressed the UUO-induced upregulation of TGF- $\beta 1$ and $\mathrm{p}-\mathrm{Smad} 2 / 3$ protein expression levels in renal tissues. Furthermore, TGF- $\beta 1$-induced activation and phosphorylation of Smad2/3 was also repressed by ASX in NRK-52E cells. These results indicated that the TGF- $\beta 1 /$ Smad signaling pathway may be involved in the protective mechanism of ASX against renal fibrosis.

In conclusion, the present study demonstrated that ASX ameliorated UUO-induced renal interstitial fibrosis in mice by increasing the number of peritubular capillaries via regulation of VEGF-A and TSP-1 expression. In addition, ASX exerted its anti-fibrotic effect by inhibiting activation of the TGF- $\beta 1 / \mathrm{Smad}$ signaling pathway. Although the detailed mechanisms must be further investigated, the results suggested that ASX could be a potential drug for alleviating renal fibrosis.

\section{Acknowledgements}

Not applicable.

\section{Funding}

The present study was supported by a grant from the Science and Technology Development Project of Xi'an Science and Technology Bureau (grant no. 2016046SF/YX02).

\section{Availability of data and materials}

All data generated or analyzed during this study are included in this published article.

\section{Authors' contributions}

$\mathrm{JZ}$ and MG were responsible for the experimental design. MM, JZ and LL performed the experiments. JZ, XZ, LZ and $\mathrm{CW}$ contributed to data analysis and drafted the manuscript. $\mathrm{JZ}$ wrote the manuscript. XZ, LZ, CW, and MG revised the manuscript. All authors read and approved the final manuscript.

\section{Ethics approval and consent to participate}

All animal protocols used in the present study were approved by the Institutional Animal Care and Use Committee of Xi'an No. 4 Hospital (Xi'an, China).

\section{Patient consent for publication}

Not applicable.

\section{Competing interests}

The authors declare that they have no competing interests.

\section{References}

1. Fang Y, Yu X, Liu Y, Kriegel AJ, Heng Y, Xu X, Liang M and Ding X: miR-29c is downregulated in renal interstitial fibrosis in humans and rats and restored by HIF-alpha activation. Am J Physiol Renal Physiol 304: F1274-F1282, 2013.

2. Boor P, Ostendorf T and Floege J: Renal fibrosis: Novel insights into mechanisms and therapeutic targets. Nat Rev Nephrol 6: 643-656, 2010.

3. Sun D, Feng J, Dai C, Sun L, Jin T, Ma J and Wang L: Role of peritubular capillary loss and hypoxia in progressive tubulointerstitial fibrosis in a rat model of aristolochic acid nephropathy. Am J Nephrol 26: 363-371, 2006.

4. Kang DH, Hughes J, Mazzali M, Schreiner GF and Johnson RJ: Impaired angiogenesis in the remnant kidney model: II. Vascular endothelial growth factor administration reduces renal fibrosis and stabilizes renal function. J Am Soc Nephrol 12: 1448-1457, 2001.

5. Babickova J, Klinkhammer BM, Buhl EM, Djudjaj S, Hoss M, Heymann F, Tacke F, Floege J, Becker JU and Boor P: Regardless of etiology, progressive renal disease causes ultrastructural and functional alterations of peritubular capillaries. Kidney Int 91: 70-85, 2017.

6. Lee JY, Song SH, Kim YS, Lim BJ, Kim SI, Kim MS and Jeong HJ: Tubuloreticular inclusions in peritubular capillaries of renal allografts. Pathol Res Pract 213: 1185-1190, 2017.

7. Boor P, Babickova J, Steegh F, Hautvast P, Martin IV, Djudjaj S, Nakagawa T, Ehling J, Gremse F, Bücher E, et al: Role of platelet-derived growth factor-CC in capillary rarefaction in renal fibrosis. Am J Pathol 185: 2132-2142, 2015.

8. Bohle A, von Gise H, Mackensen-Haen S and Stark-Jakob B: The obliteration of the postglomerular capillaries and its influence upon the function of both glomeruli and tubuli. Functional interpretation of morphologic findings. Klin Wochenschr 59: 1043-1051, 1981.

9. Fine LG, Orphanides C and Norman JT: Progressive renal disease: The chronic hypoxia hypothesis. Kidney Int (Suppl 65): S74-S78, 1998.

10. Venkatachalam MA, Weinberg JM, Kriz W and Bidani AK: Failed tubule recovery, AKI-CKD transition, and kidney disease progression. J Am Soc Nephrol 26: 1765-1776, 2015.

11. Tanaka $T$ and Nangaku M: Angiogenesis and hypoxia in the kidney. Nat Rev Nephrol 9: 211-222, 2013.

12. Kida Y, Tchao BN and Yamaguchi I: Peritubular capillary rarefaction: A new therapeutic target in chronic kidney disease. Pediatr Nephrol 29: 333-342, 2014.

13. Reinders ME, Rabelink TJ and Briscoe DM: Angiogenesis and endothelial cell repair in renal disease and allograft rejection. J Am Soc Nephrol 17: 932-942, 2006.

14. Mayer G: Capillary rarefaction, hypoxia, VEGF and angiogenesis in chronic renal disease. Nephrol Dial Transplant 26: 1132-1137, 2011.

15. Failla CM, Carbo M and Morea V: Positive and negative regulation of angiogenesis by soluble vascular endothelial growth factor receptor-1. Int J Mol Sci 19: pii: E1306, 2018. 
16. Nangaku M and Eckardt KU: Hypoxia and the HIF system in kidney disease. J Mol Med (Berl) 85: 1325-1330, 2007.

17. Hugo C: The thrombospondin 1-TGF-beta axis in fibrotic renal disease. Nephrol Dial Transplant 18: 1241-1245, 2003.

18. Xue Y, Qu Z, Fu J, Zhen J, Wang W and Cai Y: The protective effect of astaxanthin on learning and memory deficits and oxidative stress in a mouse model of repeated cerebral ischemia/reperfusion. Brain Res Bull 131: 221-228, 2017.

19. Park JS, Chyun JH, Kim YK, Line LL and Chew BP: Astaxanthin decreased oxidative stress and inflammation and enhanced immune response in humans. Nutr Metab (Lond) 7: 18, 2010.

20. Ni X, Yu H, Wang S, Zhang C and Shen S: Astaxanthin inhibits PC-3 xenograft prostate tumor growth in nude mice. Mar Drugs 15: pii: E66, 2017.

21. Komatsu T, Sasaki S, Manabe Y, Hirata T and Sugawara T: Preventive effect of dietary astaxanthin on UVA-induced skin photoaging in hairless mice. PLoS One 12: e0171178, 2017.

22. Kanazashi M, Okumura Y, Al-Nassan S, Murakami S, Kondo H, Nagatomo F, Fujita N, Ishihara A, Roy RR and Fujino H: Protective effects of astaxanthin on capillary regression in atrophied soleus muscle of rats. Acta Physiol (Oxf) 207: 405-415, 2013.

23. Liu G, Shi Y, Peng X, Liu H, Peng Y and He L: Astaxanthin attenuates adriamycin-induced focal segmental glomerulosclerosis. Pharmacology 95: 193-200, 2015.

24. Xie C, Meng M, Yin X, He F, Ye H and Xie D: Effects of astaxanthin on renal fibrosis and cell apoptosis induced by partia unilateral ureteral obstruction in rats. Nan Fang Yi Ke Da Xue Xue Bao 33: 305-308, 2013 (In Chinese).

25. Stroo I, Emal D, Butter LM, Teske GJ, Claessen N, Dessing MC Girardin SE, Florquin S and Leemans JC: No difference in renal injury and fibrosis between wild-type and NOD1/NOD2 double knockout mice with chronic kidney disease induced by uretera obstruction. BMC Nephrol 19: 78, 2018.

26. Zhao YN, Xu GJ and Yang P: GBP1 exerts inhibitory effects on acute viral myocarditis through the inhibition of inflammatory response of macrophage in mice. Biochem Cell Biol: Dec 17, 2018 (Epub ahead of print).

27. Chiang CK, Sheu ML, Lin YW, Wu CT, Yang CC, Chen MW Hung KY, Wu KD and Liu SH: Honokiol ameliorates renal fibrosis by inhibiting extracellular matrix and pro-inflammatory factors in vivo and in vitro. Br J Pharmacol 163: 586-597, 2011.

28. Chen Q, Tao J, Li G, Zheng D, Tan Y, Li R, Tian L, Li Z, Cheng $\mathrm{H}$ and $\mathrm{Xie} \mathrm{X}$ : Astaxanthin ameliorates experimental diabetes-induced renal oxidative stress and fibronectin by upregulating connexin43 in glomerular mesangial cells and diabetic mice. Eur J Pharmacol 840: 33-43, 2018.

29. Livak KJ and Schmittgen TD: Analysis of relative gene expression data using real-time quantitative PCR and the 2(-Delta Delta C(T)) method. Methods 25: 402-408, 2001

30. Liu B, Ding F, Hu D, Zhou Y, Long C, Shen L, Zhang Y, Zhang D and Wei G: Human umbilical cord mesenchymal stem cell conditioned medium attenuates renal fibrosis by reducing inflammation and epithelial-to-mesenchymal transition via the TLR4/NF- $\kappa$ B signaling pathway in vivo and in vitro. Stem Cell Res Ther 9: 7, 2018.

31. Hu N, Duan J, Li H, Wang Y, Wang F, Chu J, Sun J, Liu M, Wang C, Lu C and Wen A: Hydroxysafflor yellow a ameliorates renal fibrosis by suppressing tgf-betal-induced epithelial-to-mesenchymal transition. PLoS One 11: e0153409, 2016.

32. Zhang G, Kang Y, Zhou C, Cui R, Jia M, Hu S, Ji X, Yuan J, Cui $\mathrm{H}$ and Shi G: Amelioratory effects of testosterone propionate on age-related renal fibrosis via suppression of TGF- $\beta 1 / \mathrm{smad}$ signaling and activation of Nrf2-ARE signaling. Sci Rep 8: 10726,2018

33. Roberts V, Lu B, Chia J, Cowan PJ and Dwyer KM: CD39 overexpression does not attenuate renal fibrosis in the unilatera ureteric obstructive model of chronic kidney disease. Purinergic Signal 12: 653-660, 2016.

34. Stefanska A, Eng D, Kaverina N, Pippin JW, Gross KW, Duffield JS and Shankland SJ: Cells of renin lineage express hypoxia inducible factor 2alpha following experimental ureteral obstruction. BMC Nephrol 17: 5, 2016.

35. Liu Y: Renal fibrosis: New insights into the pathogenesis and therapeutics. Kidney Int 69: 213-217, 2006.

36. Farris AB and Colvin RB: Renal interstitial fibrosis: Mechanisms and evaluation. Curr Opin Nephrol Hypertens 21: 289-300, 2012.

37. Myllyharju J and Kivirikko KI: Collagens, modifying enzymes and their mutations in humans, flies and worms. Trends Genet 20 : 33-43, 2004.
38. Kadler K: Extracellular matrix 1: Fibril-forming collagens Protein Profile 2: 491-619, 1995.

39. Hasegawa D, Fujii R, Yagishita N, Matsumoto N, Aratani S, Izumi T, Azakami K, Nakazawa M, Fujita H, Sato T, et al: E3 ubiquitin ligase synoviolin is involved in liver fibrogenesis. PLoS One 5: e13590, 2010.

40. Holopainen I and Kontro P: Uptake and release of glycine in cerebellar granule cells and astrocytes in primary culture: Potassium-stimulated release from granule cells is calcium-dependent. J Neurosci Res 24: 374-383, 1989.

41. Lopez-Hernandez FJ and Lopez-Novoa JM: Role of TGF-beta in chronic kidney disease: An integration of tubular, glomerular and vascular effects. Cell Tissue Res 347: 141-154, 2012.

42. Chen SJ, Wu P, Sun LJ, Zhou B, Niu W, Liu S, Lin FJ and Jiang GR: miR-204 regulates epithelial-mesenchymal transition by targeting SP1 in the tubular epithelial cells after acute kidney injury induced by ischemia-reperfusion. Oncol Rep 37: 1148-1158, 2017

43. Choi HS, Song JH, Kim IJ, Joo SY1, Eom GH, Kim I, Cha H, Cho JM, Ma SK, Kim SW and Bae EH: Histone deacetylase inhibitor, CG200745 attenuates renal fibrosis in obstructive kidney disease. Sci Rep 8: 11546, 2018.

44. Nangaku M: Chronic hypoxia and tubulointerstitial injury: A final common pathway to end-stage renal failure. J Am Soc Nephrol 17: 17-25, 2006.

45. Kang DH, Anderson S, Kim YG, Mazzalli M, Suga S, Jefferson JA, Gordon KL, Oyama TT, Hughes J and Hugo C: Impaired angiogenesis in the aging kidney: Vascular endothelial growth factor and thrombospondin-1 in renal disease. Am J Kidney Dis 37: 601-611, 2001.

46. Rudnicki M, Perco P, Enrich J, Eder S, Heininger D, Bernthaler A, Wiesinger M, Sarközi R, Noppert SJ, Schramek H, et al: Hypoxia response and VEGF-A expression in human proximal tubular epithelial cells in stable and progressive renal disease. Lab Invest 89: 337-346, 2009.

47. Isenberg JS, Martin-Manso G, Maxhimer JB and Roberts DD: Regulation of nitric oxide signalling by thrombospondin 1 : implications for anti-angiogenic therapies. Nat Rev Cancer 9: 182-194, 2009.

48. Crawford SE, Stellmach V, Murphy-Ullrich JE, Ribeiro SM, Lawler J, Hynes RO, Boivin GP and Bouck N: Thrombospondin-1 is a major activator of TGF-betal in vivo. Cell 93: 1159-1170, 1998.

49. Kang DH, Kanellis J, Hugo C, Truong L, Anderson S, Kerjaschki D, Schreiner GF and Johnson RJ: Role of the microvascular endothelium in progressive renal disease. J Am Soc Nephrol 13: 806-816, 2002

50. Iruela-Arispe ML, Bornstein P and Sage H: Thrombospondin exerts an antiangiogenic effect on cord formation by endothelial cells in vitro. Proc Natl Acad Sci USA 88: 5026-5030, 1991

51. Zhang X and Lawler J: Thrombospondin-based antiangiogenic therapy. Microvasc Res 74: 90-99, 2007.

52. Sun D, Ma Y, Han H, Yin Z, Liu C, Feng J, Zhou X, Li X, Xiao A and Yu R: Thrombospondin-1 short hairpin RNA suppresses tubulointerstitial fibrosis in the kidney of ureteral obstruction by ameliorating peritubular capillary injury. Kidney Blood Press Res 35: 35-47, 2012.

53. Liao F, Li G, Yuan W, Chen Y, Zuo Y, Rashid K, Zhang JH, Feng $\mathrm{H}$ and Liu F: LSKL peptide alleviates subarachnoid fibrosis and hydrocephalus by inhibiting TSP1-mediated TGF- $\beta 1$ signaling activity following subarachnoid hemorrhage in rats. Exp Ther Med 12: 2537-2543, 2016.

54. Sun H, Zhao Y, Bi X, Li S, Su G, Miao Y, Ma X, Zhang Y, Zhang W and Zhong M: Valsartan blocks thrombospondin/transforming growth factor/Smads to inhibit aortic remodeling in diabetic rats. Diagn Pathol 10: 18, 2015.

55. Zeisberg M, Tampe B, LeBleu V, Tampe D, Zeisberg EM and Kalluri R: Thrombospondin-1 deficiency causes a shift from fibroproliferative to inflammatory kidney disease and delays onset of renal failure. Am J Pathol 184: 2687-2698, 2014.

56. Ma L, Li H, Zhang S, Xiong X, Chen K, Jiang P, Jiang K and Deng G: Emodin ameliorates renal fibrosis in rats via TGF- $\beta 1 /$ Smad signaling pathway and function study of Smurf 2. Int Urol Nephrol 50: 373-382, 2018.

57. Loeffler I and Wolf G: Epithelial-to-mesenchymal transition in diabetic nephropathy: Fact or Fiction? Cells 4: 631-652, 2015

58. Schiller M, Javelaud D and Mauviel A: TGF-beta-induced SMAD signaling and gene regulation: Consequences for extracellular matrix remodeling and wound healing. J Dermatol Sci 35: 83-92, 2004. 
59. Lan HY: Smads as therapeutic targets for chronic kidney disease. Kidney Res Clin Pract 31: 4-11, 2012.

60. Loeffler I, Liebisch M, Allert S, Kunisch E, Kinne RW and Wolf G: FSP1-specific SMAD2 knockout in renal tubular, endothelial, and interstitial cells reduces fibrosis and epithelial-to-mesenchymal transition in murine STZ-induced diabetic nephropathy. Cell Tissue Res 372: 115-133, 2018.

61. Chung AC, Zhang H, Kong YZ, Tan JJ, Huang XR, Kopp JB and Lan HY: Advanced glycation end-products induce tubular CTGF via TGF-beta-independent Smad3 signaling. J Am Soc Nephrol 21: 249-260, 2010
62. Fujimoto M, Maezawa Y, Yokote K, Joh K, Kobayashi K, Kawamura H, Nishimura M, Roberts AB, Saito Y and Mori S: Mice lacking Smad3 are protected against streptozotocin-induced diabetic glomerulopathy. Biochem Biophys Res Commun 305: 1002-1007, 2003.

63. Li J, Qu X, Yao J, Caruana G, Ricardo SD, Yamamoto Y, Yamamoto $\mathrm{H}$ and Bertram JF: Blockade of endothelial-mesenchymal transition by a Smad3 inhibitor delays the early development of streptozotocin-induced diabetic nephropathy. Diabetes 59: 2612-2624, 2010.

(i) (2) This work is licensed under a Creative Commons Attribution-NonCommercial-NoDerivatives 4.0 International (CC BY-NC-ND 4.0) License. 